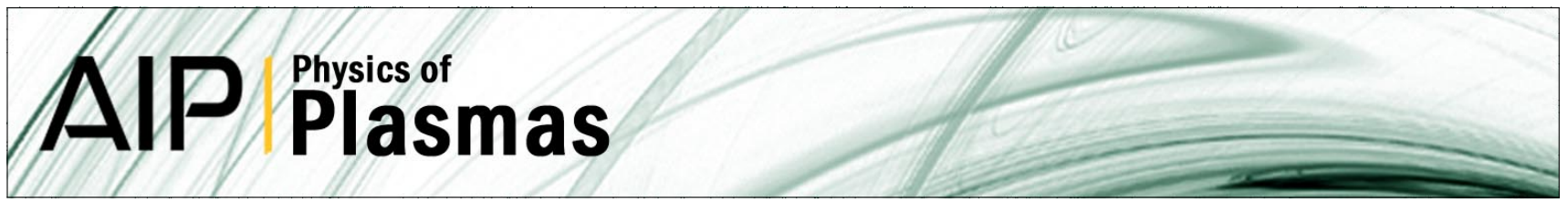

\title{
Secondary instability as cause of minor disruptions in density limit tokamak plasmas
}

F. Salzedas

Citation: Phys. Plasmas 18, 100701 (2011); doi: 10.1063/1.3644475

View online: http://dx.doi.org/10.1063/1.3644475

View Table of Contents: http://pop.aip.org/resource/1/PHPAEN/v18/i10

Published by the American Institute of Physics.

\section{Related Articles}

Fully kinetic description of the linear excitation and nonlinear saturation of fast-ion-driven geodesic acoustic mode instability

Phys. Plasmas 19, 022102 (2012)

Effects of magnetic shear on magneto-Rayleigh-Taylor instability

Phys. Plasmas 19, 022703 (2012)

Dynamic mitigation of instabilities

Phys. Plasmas 19, 024503 (2012)

Reduced magnetohydrodynamic theory of oblique plasmoid instabilities

Phys. Plasmas 19, 022101 (2012)

Magnetohydrodynamic instabilities in radial foil configurations

Phys. Plasmas 19, 022701 (2012)

\section{Additional information on Phys. Plasmas}

Journal Homepage: http://pop.aip.org/

Journal Information: http://pop.aip.org/about/about_the_journal

Top downloads: http://pop.aip.org/features/most_downloaded

Information for Authors: http://pop.aip.org/authors

\section{ADVERTISEMENT}

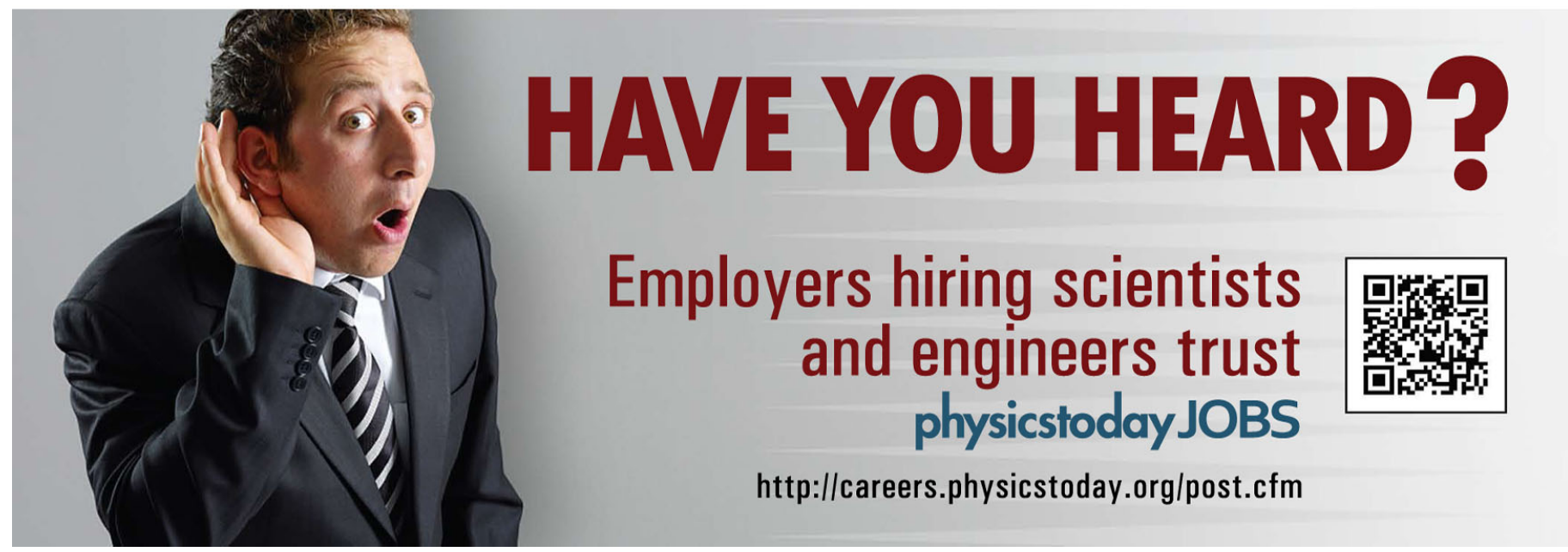




\title{
Secondary instability as cause of minor disruptions in density limit tokamak plasmas
}

\author{
F. Salzedas ${ }^{1,2,3}$ and JET EFDA Contributors ${ }^{1, a)}$ \\ ${ }^{1}$ JET EFDA, Culham Science Centre, Abingdon, OX14 3DB, United Kingdom \\ ${ }^{2}$ Eng. Phys. Department, Faculdade de Engenharia da Universidade do Porto, Rua Roberto Frias $s / n$, \\ 4200-465 Porto, Portugal \\ ${ }^{3}$ Instituto de Plasmas e Fusão Nuclear, Association Euratom-IST, Av. Rovisco Pais, 1049-001 Lisboa, \\ Portugal
}

(Received 20 June 2011; accepted 30 August 2011; published online 4 October 2011)

\begin{abstract}
Experimental evidence was found in JET plasmas of a new instability at the onset of minor disruptions. This instability is observed during the growth of the well known $m / n=2 / 1$ magnetic island and is localized close to it, behaving as a secondary instability to the island. The large heat fluxes towards the plasma edge, characteristic of minor disruptions, occur during the low rotation phase of the magnetic island at a time the amplitude of the secondary instability suffers a large increase. No poloidal or toroidal mode numbers could be assigned to the secondary instability. [doi:10.1063/1.3644475]
\end{abstract}

Major disruptions are the most dangerous instabilities in tokamak plasmas. They completely destroy the confinement of plasma thermal energy and plasma current. High fusion yield plasmas have a parameter space prone to disruptions either because they need to have high density or because they need a radiating mantle to relieve the heat load from the divertor. Understanding the cause and dynamics of major disruptions will improve the strategies to avoid or ameliorate their effects, which is a relevant issue for ITER. A well established $^{1,2}$ sequence of events in the precursor of density limit disruptions starts with the radiative contraction of the current profile caused by increase of electron density with impurity accumulation in the edge. Contraction of the current profile follows, destabilizing MHD modes, mainly an $m / n=2 / 1$ tearing mode (where $m$ and $n$ are the poloidal and toroidal Fourier mode numbers, respectively). During the growth of this magnetic island, minor disruptions may be observed before the major disruption occurs.

Minor disruptions are characterized by a sudden large heat flux across the $q=2$ surface towards the plasma edge. ${ }^{1}$ The adjective minor refers to the fact that only a fraction of the plasma energy is lost and the plasma current is not affected. The plasma can recover from minor disruptions but if no action is taken to control the evolution of the island at $q=2$, a major disruption will end the plasma. A question that has received attention is the cause of the sudden heat flux to the plasma edge. A well known proposal to answer this question is based on the interaction between MHD tearing modes at different rational surfaces from $q=1$ to $q=2$. Island overlapping is expected to provoke ergodization of magnetic field lines increasing radial transport. Despite the calculated ergodic magnetic field is a vacuum field, it is assumed that the same field will also occur in the plasma. At odds with this proposal are observations of minor disruptions with nonover-

\footnotetext{
${ }^{\text {a) }}$ See appendix, F. Romanelli et al., in Proceedings of the 23rd IAEA Fusion EnergyConference 2010 (Daejeon, Korea); Electronic mail: fsal@fe.up.pt.
}

lapping $2 / 1$ and $1 / 1$ modes in the plasma. Also, heat flux across the $q=2$ surface is asymmetric in relation to the $\mathrm{X}$ point and it is intermittent. Moreover in the region $1 \leq q \leq 2$, large electron temperature gradients are observed during the minor disruption close to $q=1$ indicating that energy confinement is locally very high.

This paper will address the questions raised by these observations, namely why a large magnetic island only destroys energy confinement intermittently and asymmetrically. The experimental observation of a secondary instability (SI) to the magnetic island is proposed as the cause of minor disruptions. Magnetic islands with high $m, n$ values have been observed close to the $2 / 1$ island elsewhere. ${ }^{3,4}$ As will be shown ahead, no mode numbers could be assigned to the secondary instability here reported, that also has a totally different nature than the secondary structure created with a dynamic ergodic divertor reported in Ref. 5. An example limiter plasma will be used however, the events described here are all observed in similar Joint European Torus (JET) plasmas with the following typical parameters: main gas Deuterium, plasma current $1.5 \mathrm{MA} \leq I_{p} \leq 1.95 \mathrm{MA}$, toroidal magnetic field $2.47 \mathrm{~T} \leq B_{\phi} \leq 3 \mathrm{~T}$, monotonic safety factor profile, electron density $1.1 \times 10^{19} \mathrm{~m}^{-3} \leq n_{e} \leq 2.15 \times 10^{19} \mathrm{~m}^{-3}$, single null and limiter plasmas. All of these plasmas show sawtooth activity in the plasma core.

An Electron Cyclotron Emission (ECE) radiometer measures the electron temperature in optically thick plasmas. With this diagnostic, the time evolution of the magnetic island width as well as its radial position can be followed. The instability is detected, for islands widths larger than the spatial resolution of the diagnostic, through the characteristic perturbations it causes on the electron temperature profile. To use this diagnostic, the electron density has to be below the ECE cut-off frequency, which was achieved with a $\mathrm{Ne}$ gas puff. The JET ECE radiometer has 96 channels. Fast time acquisition is done on a subsample of the full set of channels. ${ }^{6}$ The evolution of the electron temperature profile 


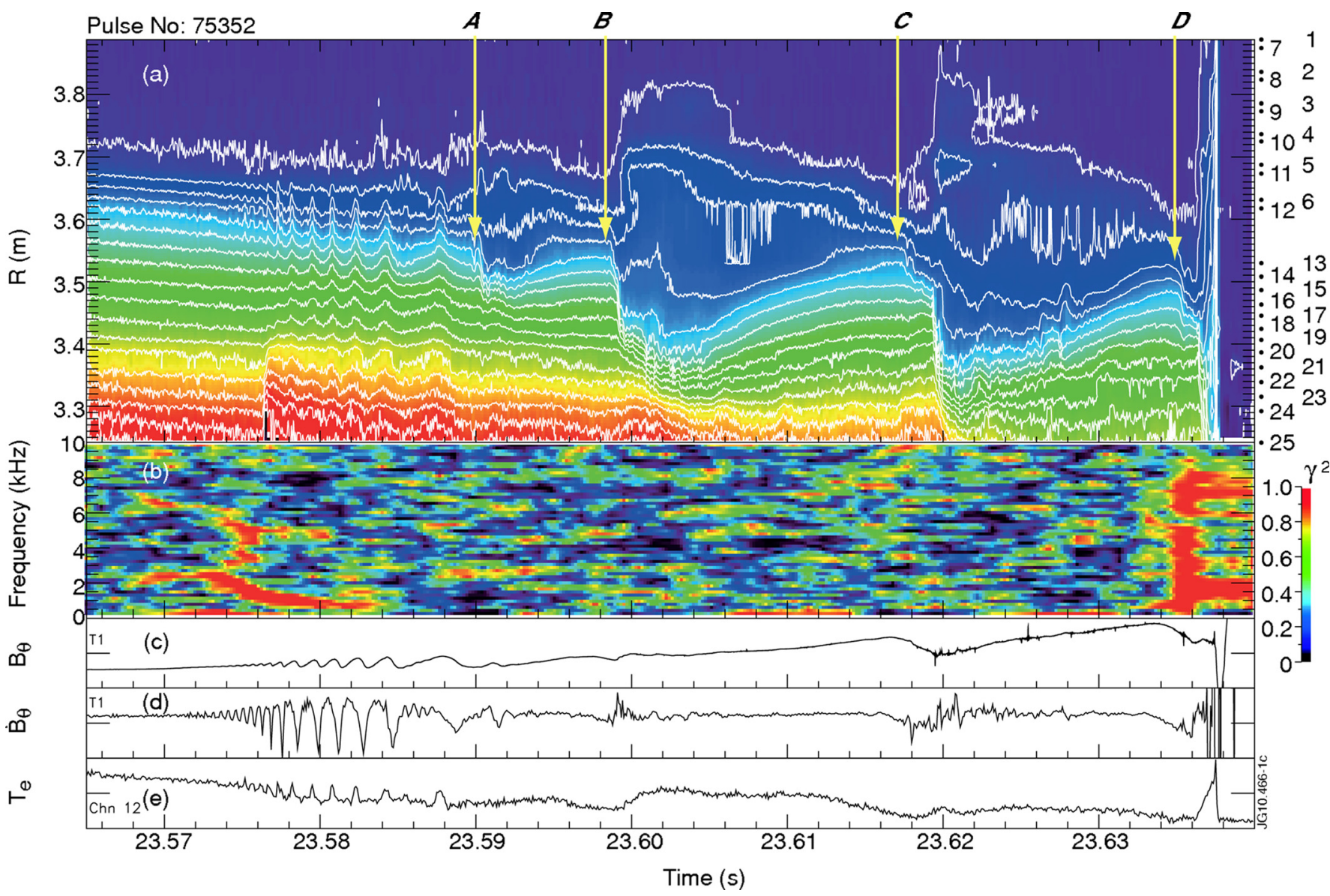

FIG. 1. (Color online) (a) ECE $T_{e}(r, t)$ isotherms. The channels position is indicated at the right. (b) Coherence between $T_{e}(t)$ and $\dot{B}_{\theta}(t)$. (c) $B_{\theta}(t)$, numerically integrated. (d) $\dot{B}_{\theta}(t)$. (e) $T_{e}(t)$. In this pulse, $I_{p}=1.7 \mathrm{MA}, q_{95}=3.5, B_{\phi}=2.7 \mathrm{~T}$, and $\bar{n}_{e}=2.15 \times 10^{19} \mathrm{~m}^{-2}$.

during the precursor of a density limit disruption is shown in Figure 1. Time resolution is $4 \mu \mathrm{s}$ and isothermals starting at $30 \mathrm{eV}$ increase in steps of $100 \mathrm{eV}$. To each channel was applied a low pass finite impulse response filter with a cut off frequency of $10 \mathrm{kHz}$ to eliminate a high frequency component that hides the observations here addressed. The figure focus on the Low Field Side (LFS) part of the profile, spanning the region from the edge to $\approx q=1$. The oscillation starting with a high frequency and small amplitude at $3.62 \mathrm{~m}$ and $23.572 \mathrm{~s}$ and evolving to low frequency and large amplitude is the signature of the 2/1 magnetic island on the LFS of the plasma. From cross-spectrum analysis, the radial position of the $q=2$ rational surface is found at $\approx 3.62 \mathrm{~m}$ (see Fig. 3). The frequency decreases nonlinearly from $2 \mathrm{kHz}$ up to $\approx 50 \mathrm{~Hz}$ before the major disruption occurs at $23.637 \mathrm{~s}$. Because the island does not lock completely to the wall, the radiometer can follow the degradation of the $T_{e}$ profile as the plasma slowly moves in front of the radiometer antenna during these events. In Figure 1(a), four distinct events with large heat fluxes across the $q=2$ initiate at $\approx 23.590 \mathrm{~s}$, $\approx 23.599 \mathrm{~s}, \approx 23.618 \mathrm{~s}$, and $\approx 23.635 \mathrm{~s}$, labeled $A, B, C$, and $D$, respectively. During these events, the heat flux is directed to the plasma edge as indicated by the increase in plasma edge temperature that follows. Edge temperature evolution also shows that heat lost during event $A$ is smaller compared with the other three events. One particular feature common to all these four events is that the sudden heat flux occurs at the same time a local oscillation secondary to the $2 / 1$ oscillation experiences an increase in amplitude. This secondary oscillation, to the magnetic island, will be called for simplicity from here on the SI. A closer observation shows that this SI is already present in the plasma before event $A$ occurs. Due to the low spatial coverage of the region around the $q=2$ surface by the fast ECE radiometer channels combined with higher noise level of the edge channels, it was difficult to follow the SI with this diagnostic during the $2 / 1$ mode fast rotating phase.

Both the 2/1 magnetic island and SI can be followed also by magnetic coils. Figure 2 (a) shows $\dot{B}_{\theta}$ signals from a toroidal array of coils ${ }^{7}$ during the fast rotating phase of the magnetic island. Two different oscillations are observed. One with large increasing amplitude and decreasing frequency (from about $2 \mathrm{kHz}$ to $100 \mathrm{~Hz}$ ) and another smaller amplitude oscillation at frequencies above $2 \mathrm{kHz}$. The gray lines connecting the minima, of the large amplitude oscillations, of the signals from different coils show that the magnetic island has a toroidal mode number $n=1$. The slope of the lines is proportional to the island average rotation frequency which decreases with time. The right most line indicates nonlinear change in toroidal rotation frequency within one complete turn of the island, with a decrease in frequency from $500 \mathrm{~Hz}$ to $130 \mathrm{~Hz}$ between $23.5855 \mathrm{~s}$ and $23.5878 \mathrm{~s}$ followed by a frequency increase to $390 \mathrm{~Hz}$ between $23.5878 \mathrm{~s}$ and $23.5888 \mathrm{~s}$. A similar exercise to find the toroidal mode 


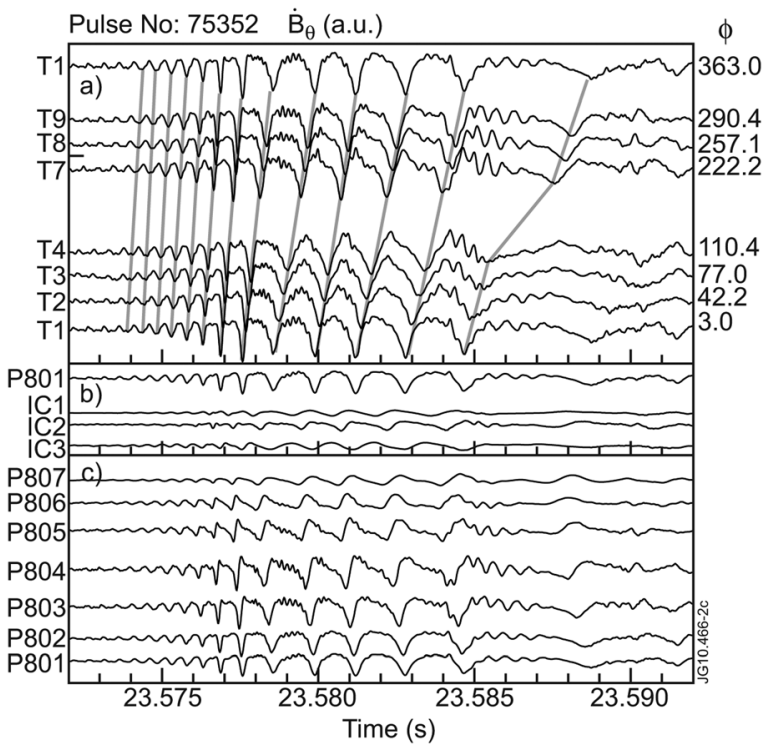

FIG. 2. (a) Toroidal array of $\dot{B}_{\theta}(t)$. Poloidal array of $\dot{B}_{\theta}(t)$ : (b) inner wall, and (c) limiter coils.

number of the SI fails as neighbor coils do not always detect the same number of minima. Moreover, the small amplitude oscillations from the SI are not seen over a complete period of the 2/1 mode oscillation. These two observations are consistent if the SI has no toroidal mode number.

Figures 2(b) and 2(c) show $\dot{B}_{\theta}$ signals from a poloidal array of coils ${ }^{7}$ during the fast rotating phase of the magnetic island. Coils IC1, IC2, and IC3 are located in the high field side (HFS) and the other seven coils are on the LFS. This array allows us to draw similar conclusions as from the toroidal array, namely the $m=2$ poloidal mode number of the magnetic island and no poloidal number for the SI. Up to $\approx 23.576 \mathrm{~s}$ the $m / n=2 / 1$ oscillations in $\dot{B}_{\theta}(t)$ show a sinusoidal pattern. Later, they became distorted coinciding with the presence of the SI. Note that coils, from the poloidal array, closer to the equatorial torus plane (P803-P805 and IC2) measure a larger distortion of the 2/1 signal as well as a larger amplitude of the SI. This distortion can be caused by the SI but other effects such as error fields, plasma flow, or a combination of these cannot be excluded so far.

As discussed previously, the SI has no toroidal or poloidal mode number, and relatively to the $2 / 1$ magnetic island this is consistent with the fact that SI is only observed at some parts of the 2/1 mode. During the fast rotating phase of the magnetic island (up to event $A$ ), the secondary oscillations are mostly observed at the island O point. The amplitude of SI increases as the magnetic island frequency decreases. Exception to this is the period starting at $23.583 \mathrm{~s}$, where from coil $\mathrm{T} 7$ and progressing to coils $\mathrm{T} 8, \mathrm{~T} 9, \mathrm{~T} 1, \ldots \mathrm{T} 4$, the secondary oscillations are observed continuously during one complete turn of the 2/1 mode around the torus. So in this period SI oscillations are seen at both the $\mathrm{O}$ point and the $\mathrm{X}$ point of the $2 / 1$ mode. This is also the period where, as described previously, the 2/1 mode experiences a pronounced nonlinear change in frequency (from $500 \mathrm{~Hz}$ to $130 \mathrm{~Hz}$ and then up to $390 \mathrm{~Hz}$ ). This behavior has been observed in all other similar discharges indicating that when the SI spans over the 2/1 tear- ing mode, this mode is dragged. Then follows event $A$ where a moderate large heat flux across the $q=2$ surface towards the plasma edge is observed. At this stage, the average rotation frequency of the island is $100 \mathrm{~Hz}$ and the SI develops in the magnetic island $O$ point (see Fig. 1(a)).

Minor disruptions, events $B, C$, and $D$, where larger heat fluxes across the $q=2$ are observed, only occur when the $2 / 1$ island rotation frequency is around $50 \mathrm{~Hz}$. At these stages, the heat flux to the edge experiences a significant increase when the SI develops a large amplitude at the magnetic island X point. Note, however, that SI is only observed in half of the 2/1 island, namely from the time between the $X$ point passes in front of the ECE radiometer $(23.598 \mathrm{~s})$ till the $\mathrm{O}$ point passes $(23.604 \mathrm{~s})$. Then from this time, until the time the $\mathrm{X}$ point passes again (23.617 s) the SI is not observed. Enhanced heat fluxes are only observed where the SI is located and when the SI oscillations are visible. In the part of the island not affected by SI, the electron temperature increases indicating good energy confinement. So, during the low rotation frequency phase, relatively to the island toroidal velocity, in the upstream direction, experimental data clearly indicate that in one part of the island (between the $\mathrm{X}$ and $\mathrm{O}$ points) the SI degrades energy confinement, while at the other part of the island (between the $\mathrm{O}$ and $\mathrm{X}$ points) without SI confinement is not affected as shown by the $T_{e}$ increase in the region between the low $q$ side of the island separatrix and $\approx q=1$.

From Figure 1, one can gain some insight about the radial location of the SI. The location of the 2/1 magnetic island separatrix is not precisely known. However, during the slow rotating phase of the 2/1 island the SI is apparently located in the vicinity of the low $q$ side of the separatrix. This is supported by the lack of secondary oscillations on $T_{e}$ at the high $q$ side of the separatrix during minor disruptions (event $B, C$, and $D$ ). During the fast rotation phase, the SI oscillations are found at the high $q$ side of the separatrix (e.g., at $23.585 \mathrm{~s}$ and at $23.590 \mathrm{~s}$ ). However, this can be due to the smaller island width at this time.

The perturbations the 2/1 island leaves on $T_{e}$ and $\dot{B}_{\theta}$ should share similar frequency spectrum. Since radial position of $\tilde{T}_{e}$ is known, position of $\dot{B}_{\theta}$ perturbations linearly dependent with $\tilde{T}_{e}$ can be inferred from the coherence function,

$$
\gamma^{2}(f)=\frac{\left|S_{T_{e} \dot{B}_{\theta}}(f)\right|^{2}}{S_{T_{e} T_{e}}(f) S_{\dot{B}_{\theta} \dot{B}_{\theta}}(f)},
$$

where $S$ is the cross-spectrum between $T_{e}$ and $\dot{B}_{\theta}$, and $0 \leq \gamma^{2}(f) \leq 1$. Coherence between the $T_{e}$ signal measured by channel 12 of the ECE radiometer (the closest channel to the $q=2$ surface) and $\dot{B}_{\theta}$ signal measured by coil T1 is shown on Figure 1(b). These two signals have high coherence between $23.57 \mathrm{~s}$ and $23.58 \mathrm{~s}$ with frequency varying from $2 \mathrm{kH}$ to $500 \mathrm{~Hz}$, which corresponds to the first $10 \mathrm{~ms}$ of growth of the $2 / 1$ mode. Around $23.575 \mathrm{~s}$ high coherence is also observed for frequencies between 4 and $6 \mathrm{kHz}$. Closer inspection of the $\dot{B}_{\theta}$ signal shows that the frequency of the SI oscillations at this time is also in the same range. So this spot of high coherence between the two signals can be attributed 


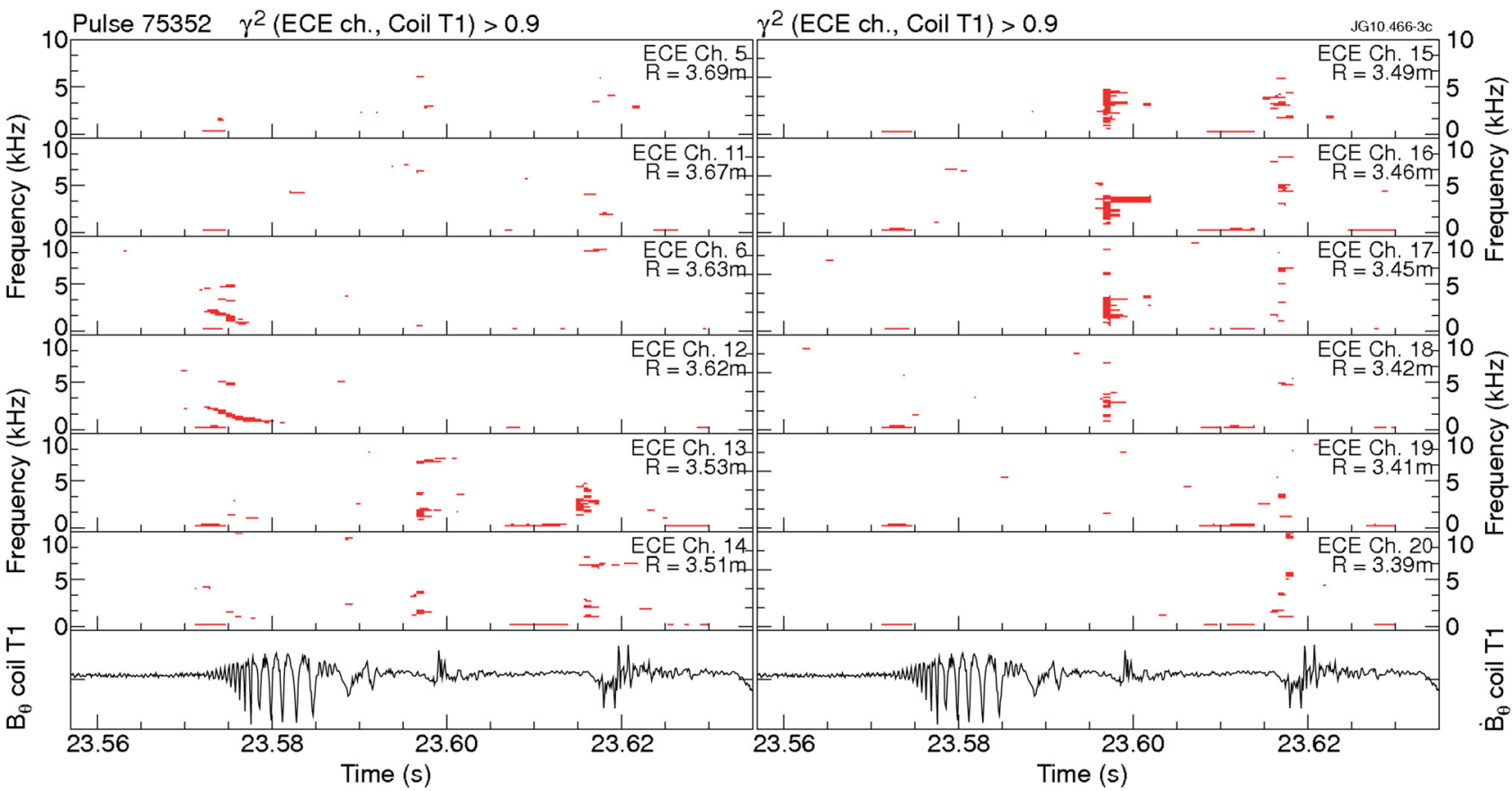

FIG. 3. (Color online) (a) Coherence between $T_{e}$ and $\dot{B}_{\theta}(t)$ for the indicated radiometer channels. For reference, $\dot{B}_{\theta}(t)$ is shown at the bottom of each column.

to the SI. The two signals will only show high coherence again at the major disruption.

High coherence values, $\gamma^{2}>0.9$, between the signals of several ECE channels and the signal from T1 coil is represented in Figure 3. It is observed for ECE channels from 13 to 18 , high coherence with the $\dot{B}_{\theta}$ signal of coil $\mathrm{T} 1$ at the minor disruptions (events $\mathrm{B}$ and $\mathrm{C}$ ). The magnetic island at these times is rotating at very low frequencies $(\approx 50 \mathrm{~Hz})$, so the high coherence values occurring are most probably due to the higher frequency oscillations of the SI. The other channels $(19,20, \ldots$ up to plasma core and $12,6, \ldots$ up to the edge) do not have high coherence with $\dot{B}_{\theta}$ from coil T1. This corroborates Fig. 1(a), where the SI is seen in the region close to the low $q$ side magnetic island separatrix during the minor disruptions ( $\mathrm{B}, \mathrm{C}$, and $\mathrm{D})$.

The SI during event A shows similar features with a predisruptive instability observed in RTP, a tokamak with a minor radius one order of magnitude smaller than JET (see arrow A in Figures 2 and 4 of Ref. 8). Also in RTP, once the disturbance of $T_{e}$ caused by the instability is observed, the sinusoidal $\dot{B}_{\theta}$ signal became distorted. The time scales differ quite a lot between the two tokamaks and very likely in JET, with longer time scales, the SI here described is the same instability observed in RTP, but seen in more detail and during a longer period. If that proves to be the case, the SI is not simply a machine dependent instability but a universal one that will certainly be seen in other tokamaks and in ITER.

This work was performed under the Euratom-IST Association agreement, with financial support from Euratom and was carried out within the framework of the European
Fusion Development Agreement. The views and opinions expressed herein do not necessarily reflect those of the European Commission.

${ }^{1}$ S. Mirnov, J. Wesley, N. Fujisawa, Yu. Gribov, O. Gruber, T. Hender, N. Ivanov, S. Jardin, J. Lister, F. Perkins, M. Rosenbluth, N. Sauthoff, T. Taylor, S. Tokuda, K. Yamazaki, R. Yoshino, A. Bondeson, J. Conner, E. Fredrickson, D. Gates, R. Granetz, R. La Haye, J. Neuhauser, F. Porcelli, F. W. Perkins, D. E. Post, N. A. Uckan, M. Azumi, D. J. Campbell, N. Ivanov, M. Wakatani, W. M. Nevins, M. Shimada, and J. Van Dan, Nucl. Fusion 39, 2251 (1999).

${ }^{2}$ T. C. Hender, J. C. Wesley, J. Bialek, A. Bondeson, A. H. Boozer, R. J. Buttery, A. Garofalo, T. P. Goodman, R. S. Granetz, Y. Gribov, O. Gruber, M. Gryaznevich, G. Giruzzi, S. Gunter, N. Hayashi, P. Helander, C. C. Hegna, D. F. Howell, D. A. Humphreys, G. T. A. Huysmans, A. W. Hyatt, A. Isayama, S. C. Jardin, Y. Kawano, A. Kellman, C. Kessel, H. R. Koslowski, R. J. La Haye, E. Lazzaro, Y. Q. Liu, V. Lukash, J. Manickam, S. Medvedev, V. Mertens, S. V. Mirnov, Y. Nakamura, G. Navratil, M. Okabayashi, T. Ozeki, R. Paccagnella, G. Pautasso, F. Porcelli, V. D. Pustovitov, V. Riccardo, M. Sato, O. Sauter, M. J. Schaffer, M. Shimada, P. Sonato, E. J. Strait, M. Sugihara, M. Takechi, A. D. Turnbull, E. Westerhof, D. G. Whyte, R. Yoshino, H. Zohm and the ITPA MHD, Disruption and Magnetic Control Topical Group, Nucl. Fusion 47, S128 (2007).

${ }^{3}$ A. J. H. Donné, J.C. van Gorkom, V. S. Udintsev, C. W. Domier, A. Krämer-Flecken, N. C. Luhmann, and F. C. Schüller, Phys. Rev. Lett. 94, 085001 (2005).

${ }^{4}$ H. Tojo, Ph.D. dissertation (University of Tokyo, 2009), URL: http://hdl. handle.net/2261/32657.

${ }^{5}$ Y. Liang, H. R. Koslowski, A. Krämer-Flecken, O. Zimmermann, K. Löwenbrück, G. Bertschinger, and R. C. Wolf, Nucl. Fusion 47, L21 (2007).

${ }^{6}$ E. de la Luna, J. Sánchez, V. Tribaldos, JET-EFDA contributors, G. Conway, W. Suttrop, J. Fessey, R. Prentice, C. Gowers, J.M. Chareau, Rev. Sci. Instrum. 75, 3831 (2004).

${ }^{7}$ M. F. F. Nave, D. Borba, R. Galvão, S. Hacquin, B. Alper, C. Challis, S. Gerasimov, N. Hawkes, J. Mailloux, S. Sharapov, C. Boswell, M. Brix, E. Joffrin, E. de la Luna, P. Smeulders, EFDA-JET Contributors, Rev. Sci. Instrum. 75, 4247 (2004).

${ }^{8}$ F. Salzedas, S. Hokin, F. C. Schüller, and A. A. M. Oomens, Phys. Plasmas 9, 3402 (2002). 